\title{
Singularities and stable homotopy groups of spheres. I
}

\author{
by \\ Csaba Nagy, András Szűcs and Tamás Terpai
}

\begin{abstract}
We establish an interesting connection between Morin singularities and stable homotopy groups of spheres. We apply this connection to computations of cobordism groups of certain singular maps. The differentials of the spectral sequence computing these cobordism groups are given by the composition multiplication in the stable homotopy groups of spheres.
\end{abstract}

\section{$\S 1 \quad$ Introduction}

We are considering stable smooth maps of $n$-dimensional manifolds into $(n+1)$-dimensional manifolds with the simplest, corank 1 singularities (those where the rank of the differential map is at most 1 less than the maximum possible). These singularities, called Morin singularities, form a single infinite family, with members denoted by symbols $\Sigma^{0}$ (nonsingular points), $\Sigma^{1,0}$ (fold points), $\Sigma^{1,1,0}=\Sigma^{1_{2}}$ (cusp points), .., $\Sigma^{1, \ldots, 1,0}=\Sigma^{1_{j}}, \ldots$ (see [M]).

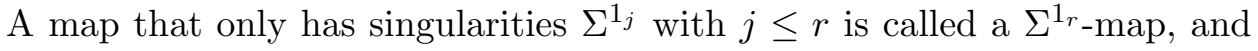
we are interested in calculating the cobordism groups of such maps. Two $\Sigma^{1_{r}}$-maps with the same target manifold $P$ are $\left(\Sigma^{1_{r}}-\right)$ cobordant if there exists a $\Sigma^{1_{r}}$-map from another manifold with boundary into $P \times[0,1]$ whose boundary is the disjoint union of those two maps. Unless specified otherwise maps between manifolds will be assumed to be cooriented, Morin and of codimension 1 (that is, the dimension of the target is 1 greater than the dimension of the source).

The cobordism group of fold maps of oriented $n$-manifolds into $\mathbb{R}^{n+1}$ denoted by $\mathrm{Cob}^{S O} \Sigma^{1,0}(n)$ - was computed in [Sz5] completely, while that of cusp maps, $\mathrm{Cob}^{S O} \Sigma^{1,1}(n)$, only modulo 2- and 3-torsion.. Here we compute the 3 -torsion part (up to a group extension). We shall also consider 
a subclass of such maps, the so-called prim (projection of immersion) cusp maps. These are the cusp maps with trivial and trivialized kernel bundle of the differential over the set of singular points. The cobordism group of prim fold and cusp maps of oriented $n$-manifolds to $\mathbb{R}^{n+1}$ will be denoted by $\operatorname{Prim}^{S O} \Sigma^{1,0}(n)$ and $\operatorname{Prim}^{S O} \Sigma^{1,1}(n)$ respectively. We shall compute these groups as well.

\section{$\S 2$ Notation and formulation of the result}

We shall denote by $\pi^{s}(n)$ the $n$th stem, that is,

$$
\pi^{s}(n)=\lim _{q \rightarrow \infty} \pi_{n+q}\left(S^{q}\right)
$$

Denote by $\mathcal{G}$ the direct sum $\bigoplus_{n} \pi^{s}(n)$. Recall that $\mathcal{G}$ is a ring, with multiplication $\circ$ defined by the composition (see [To]). This product is skew commutative: for homogeneous elements $x$ and $y$ of $\mathcal{G}$

$$
x \circ y=(-1)^{\operatorname{dim} x \cdot \operatorname{dim} y} y \circ x .
$$

Recall that $\pi^{s}(3) \cong \mathbb{Z}_{3} \oplus \mathbb{Z}_{8}$; the standard notation for the generator of the $\mathbb{Z}_{3}$ part is $\alpha_{1}$. By a slight abuse of notation we shall also denote by $\alpha_{1}$ the group homomorphism of $\mathcal{G}$ defined by left multiplication by $\alpha_{1}$, i.e. $\alpha_{1}(g)=\alpha_{1} \circ g$ for any $g \in \mathcal{G}$.

To formulate our results more compactly, we use the language of Serre classes of abelian groups $[\mathrm{S}]$. In particular, we will denote by $\mathcal{C}_{2}$ the class of finite 2-torsion groups and $\mathcal{C}_{\{2,3\}}$ will denote the class of finite groups of order a product of powers of 2 and 3 . Given a Serre class $\mathfrak{C}$, we call a homomorphism $f: A \rightarrow B$ a $\mathfrak{C}$-isomorphism if both ker $f \in \mathfrak{C}$ and Coker $f \in$ $\mathfrak{C}$. Two groups are considered to be isomorphic modulo $\mathfrak{C}$ (denoted by $\underset{\mathfrak{C}}{\cong}$ ) if there exists a chain of $\mathfrak{C}$-isomorphisms that connects them. For example, isomorphism modulo $\mathcal{C}_{\{2,3\}}$ is isomorphism modulo the 2-primary and 3primary torsions. A complex ... $\stackrel{d_{m+1}}{\rightarrow} C_{m} \stackrel{d_{m}}{\rightarrow} \ldots$ of maps is called $\mathfrak{C}$-exact if both $\operatorname{im}\left(d_{m} \circ d_{m+1}\right)$ and $\operatorname{ker} d_{m} /\left(\operatorname{ker} d_{m} \cap \operatorname{im} d_{m+1}\right)$ belong to $\mathfrak{C}$ for all $m$.

Theorem 1. There is a $\mathcal{C}_{2}$-exact sequence

$$
\begin{aligned}
0 \rightarrow \operatorname{Coker}\left(\alpha_{1}: \pi^{s}(n-3)\right. & \left.\rightarrow \pi^{s}(n)\right) \rightarrow \mathrm{Cob}^{S O} \Sigma^{1,1}(n) \rightarrow \\
& \rightarrow \operatorname{ker}\left(\alpha_{1}: \pi^{s}(n-4) \rightarrow \pi^{s}(n-1)\right) \rightarrow 0
\end{aligned}
$$


Remark. Recall that in [Sz5] it was shown that

$$
\mathrm{Cob}^{S O} \Sigma^{1,1}(n) \underset{\mathcal{C}_{\{2,3\}}}{\cong} \pi^{s}(n) \oplus \pi^{s}(n-4)
$$

(in particular $\mathrm{Cob}^{S O^{1,1}}(n)$ is finite unless $n=0$ or $n=4$, when its rank is 1 ). Since $\alpha_{1}$ is a homomorphism of order 3 , Theorem 1 is compatible with this result.

Remark. Recall the first few groups $\pi^{s}(n)$ and the standard names of the generators of the 3-primary components:

\begin{tabular}{l|c|c|c|c|c|c|c|c|c|c|c|c}
$n$ & 0 & 1 & 2 & 3 & 4 & 5 & 6 & 7 & 8 & 9 & 10 & 11 \\
\hline$\pi^{s}(n)$ & $\mathbb{Z}$ & $\mathbb{Z}_{2}$ & $\mathbb{Z}_{2}$ & $\mathbb{Z}_{24}$ & 0 & 0 & $\mathbb{Z}_{2}$ & $\mathbb{Z}_{240}$ & $\mathbb{Z}_{2}^{2}$ & $\mathbb{Z}_{2}^{3}$ & $\mathbb{Z}_{6}$ & $\mathbb{Z}_{56} \times \mathbb{Z}_{9}$ \\
\hline$\pi^{s}(n)_{3}$ & $\mathbb{Z}$ & & & $\mathbb{Z}_{3}\left\langle\alpha_{1}\right\rangle$ & & & & $\mathbb{Z}_{3}\left\langle\alpha_{2}\right\rangle$ & & & $\mathbb{Z}_{3}\left\langle\beta_{1}\right\rangle$ & $\mathbb{Z}_{9}\left\langle\alpha_{3}\right\rangle$
\end{tabular}

Here and later we denote by $G_{3}$ the 3 -primary part 11 of the abelian group $G$.

For $n \leq 11$ the only occasion when the homomorphism $\alpha_{1}$ is nontrivial on the 3-primary part is the epimorphism $\pi^{s}(0) \rightarrow \pi^{s}(3)$. Hence $\mathrm{Cob}^{S O} \Sigma^{1,1}(n)_{3} \cong \pi^{s}(n)_{3} \oplus \pi^{s}(n-4)_{3}$ for $n \leq 11$ (in each case only one of the summands is non-trivial).

\section{$\S 3 \quad$ Elements of stable homotopy groups of spheres arising from singularities}

\section{$\S 3.1 \quad$ Representing elements of $\mathcal{G}$}

The following is a well-known corollary of the combination of the PontryaginThom isomorphism and the Smale-Hirsch immersion theory:

Fact 1. The cobordism group of framed immersions of $n$-manifolds into $\mathbb{R}^{n+k}$ is isomorphic to $\pi^{s}(n)$ for any $k \geq 1$.

An equivalent formulation is the following:

Fact 2. The cobordism group of pairs $\left(M^{n}, F\right)$, where $M^{n}$ is a stably parallelizable $n$-manifold and $F$ is a trivialization of its stable normal bundle is isomorphic to $\pi^{s}(n)$.

These Facts follow from the so-called (Multi-)Compression Theorem:

Theorem 2 ([RS]). a) Given an embedding $i: M^{m} \hookrightarrow \mathbb{R}^{n} \times \mathbb{R}^{k}$ of a compact manifold $M$ (possibly with boundary) equipped with $k$ linearly independent normal vector fields $v_{1}, \ldots, v_{k}$, such that $n>m$, there is an

\footnotetext{
${ }^{1}$ the quotient by the subgroup of torsion elements with orders coprime to 3
} 
isotopy $\Phi_{t}$ of $\mathbb{R}^{n} \times \mathbb{R}^{k}$ such that $\Phi_{0}$ is the identity and $d \Phi_{1}\left(v_{j}\right)$ is parallel to the $j$ th coordinate vector $e_{j}$ of $\mathbb{R}^{k}$. The same statement stays true if $n=m$ and $M$ has no closed components.

b) If some of the vector fields $v_{1}, \ldots, v_{k}$ were already parallel to the corresponding vectors $e_{1}, \ldots, e_{k}$, then the isotopy $\Phi$ can be chosen to keep them parallel at all times.

c) The isotopy $\Phi$ can be chosen in such a way that for any point $p \in M$ and any time $t_{0} \in[0,1]$ the tangent vector of the curve $t \mapsto \Phi_{t}(i(p))$ at the point $\Phi_{t_{0}}(i(p))$ will not be tangent to the manifold $\Phi_{t_{0}}(i(M))$. That is, the map $M \times[0,1] \rightarrow \mathbb{R}^{n} \times \mathbb{R}^{k}$ defined by the formula $(p, t) \mapsto \Phi_{t}(i(p))$ is an immersion.

The part $c$ ) of the theorem is not stated explicitly in [RS], but the construction given in its proof satisfies it.

Before formulating a corollary that we shall use, note that given an immersion $i: M^{m} \rightarrow \mathbb{R}^{n} \times \mathbb{R}^{k}$ with $k$ independent normal vector fields $v_{1}$, $\ldots, v_{k}$, one can consider an extension of $i$ to $M \times D_{\varepsilon}^{k}$ for a small $k$-disc $D_{\varepsilon}^{k}=\left\{\left(y_{1}, \ldots, y_{k}\right): \sum y_{j}^{2}<\varepsilon\right\}$ for any sufficiently small positive $\varepsilon$ :

$$
\begin{aligned}
\hat{i}: M \times D_{\varepsilon}^{k} & \rightarrow \mathbb{R}^{n} \times \mathbb{R}^{k} \\
\left(p, y_{1}, \ldots, y_{k}\right) & \mapsto i(p)+\sum_{j=0}^{k} y_{j} v_{j}(p)
\end{aligned}
$$

Corollary. Given an immersion $i: M^{m} \rightarrow \mathbb{R}^{n} \times \mathbb{R}^{k}$ with $k$ independent normal vector fields $v_{1}, \ldots, v_{k}$ that satisfies $m<n$ or where $M$ has no closed components and $m=n$, there is a regular homotopy $\Phi_{t}: M \times D_{\varepsilon}^{k} \rightarrow \mathbb{R}^{n} \times \mathbb{R}^{k}$ such that $\Phi_{0}=\hat{i}$ and $d \Phi_{1}\left(v_{j}\right)$ is parallel to the $j$ th coordinate vector $e_{j}$ of $\mathbb{R}^{k}$.

Proof. Lift $\hat{i}$ to an embedding $\tilde{i}: M \times D_{\varepsilon}^{k} \hookrightarrow \mathbb{R}^{n} \times \mathbb{R}^{k} \times \mathbb{R}^{N}$, adding normal vectors $u_{1}, \ldots, u_{N}$ parallel to the coordinate vectors of $\mathbb{R}^{N}$. Applying parts $a$ ) and $b$ ) of Theorem 2 to $\tilde{i}$ we obtain an isotopy $\tilde{\Phi}_{t}$ that keeps $u_{1}, \ldots, u_{N}$ parallel to their original direction. Then the projection of the isotopy $\tilde{\Phi}_{t} \circ \tilde{i}$ to $\mathbb{R}^{n} \times \mathbb{R}^{k}$ restricted to $M \times \mathbf{0}$ yields the required regular homotopy.

\section{§3.2 Framed immersions on the boundary of a singularity}

We are going to establish a connection between singularities and stable homotopy groups of spheres. Namely, we define elements of $\mathcal{G}$ that describe incidence of the images of singularity strata. 
Example 1: Let us consider the Whitney umbrella map

$$
\sigma_{1}: \mathbb{R}^{2} \rightarrow \mathbb{R}^{3}, \quad \sigma_{1}(t, x)=\left(t, t x, x^{2}\right) .
$$

(the normal form of an isolated $\Sigma^{1,0}$-point). The preimage of the unit 2sphere $S^{2} \subset \mathbb{R}^{3}$ is a closed curve $\sigma_{1}^{-1}\left(S^{2}\right)$. The restriction of $\sigma_{1}$ to this curve - called the link of the map $\sigma_{1}$ and denoted by $\partial \sigma_{1}$ - is an immersion. The image $\sigma_{1}\left(\sigma_{1}^{-1}\left(S^{2}\right)\right)$ is an immersed curve in $S^{2}$ with a single double point. The orientations of $\mathbb{R}^{2}$ and $\mathbb{R}^{3}$ give a coorientation on this curve. Hence this curve can be equipped with a normal vector in $S^{2}$ and an additional normal vector to $S^{2}$ in $\mathbb{R}^{3}$, resulting in an immersed framed curve in $\mathbb{R}^{3}$; this represents an element of $\pi^{s}(1)$ that we shall denote by $d^{1} \sigma_{1}$. It is easy to see that $d^{1} \sigma_{1} \neq 0$ (because this immersed curve in $S^{2}$ has a single double point). Using the standard notation $\eta$ for the generator of $\pi^{s}(1)=\mathbb{Z}_{2}$, we get that $d^{1} \sigma_{1}=\eta$.

Example 2: Let us consider the normal form of an isolated cusp-point

$$
\begin{aligned}
\sigma_{2}: \mathbb{R}^{4} & \rightarrow \mathbb{R}^{5} \\
\left(t_{1}, t_{2}, t_{3}, x\right) & \mapsto\left(t_{1}, t_{2}, t_{3}, z_{1}, z_{2}\right) \\
z_{1} & =t_{1} x+t_{2} x^{2} \\
z_{2} & =t_{3} x+x^{3}
\end{aligned}
$$

The link of this map is its restriction to $\sigma_{2}^{-1}\left(S^{4}\right)$, where $S^{4}$ is the unit sphere in $\mathbb{R}^{5}$. Note that $\sigma_{2}^{-1}\left(S^{4}\right)$, which we shall denote by $L^{3}$, is diffeomorphic to $S^{3}$. The link map $\partial \sigma_{2}=\left.\sigma_{2}\right|_{L^{3}}: L^{3} \rightarrow S^{4}$ is a fold map, it has only $\Sigma^{1,0}$ singularities along a closed curve $\gamma$. The image of this curve $\gamma$ in $S^{4}$ has a canonical framing. Indeed, the map $\sigma_{2}$ can be lifted to an embedding $\hat{\sigma}_{2}$ : $\mathbb{R}^{4} \hookrightarrow \mathbb{R}^{6}, \hat{\sigma}_{2}\left(t_{1}, t_{2}, t_{3}, x\right)=\left(\sigma_{2}\left(t_{1}, t_{2}, t_{3}, x\right), x\right)$ such that the composition of $\hat{\sigma}_{2}$ with the projection $\mathbb{R}^{6} \rightarrow \mathbb{R}^{5}$ is $\sigma_{2}$. Hence the two preimages of any double point of $\sigma_{2}$ near the singularity curve $\sigma_{2}(\gamma)$ have an ordering and so one gets two of the normal framing vectors on the singularity curve $\sigma_{2}(\gamma)$. In order to get the third framing vector we note that $\sigma_{2}(\gamma)$ is the boundary of the surface formed by the double points of $\partial \sigma_{2}$ in $S^{4}$. The inward-pointing normal vector along $\sigma_{2}(\gamma)$ of this surface will be the third framing vector. (In Appendix 2 we shall describe the framing that arises naturally on the image of the top singularity stratum of a map obtained as a generic projection of an immersion.) The curve $\sigma_{2}(\gamma)$ with this framing represents an element in $\pi^{s}(1)$ that we denote by $d^{1}\left(\sigma_{2}\right)$. We shall show that $d^{1}\left(\sigma_{2}\right)=0$.

In the present situation we can construct one more element of $\mathcal{G}$ associated to $\sigma_{2}$, which we shall denote by $d^{2}\left(\sigma_{2}\right)$. We construct this element 
(after making some choices) in $\pi^{s}(3) \cong \mathbb{Z}_{24}$ but it will be well-defined only in the quotient group $\mathbb{Z}_{24} / \mathbb{Z}_{2}$. The definition of $d^{2}\left(\sigma_{2}\right)$ is the following. By a result of [Sz4] (that we shall recall in $\S 5$, see also [Te]) a cobordism of the singularity curve $\sigma_{2}(\gamma)$ can be extended to a cobordism of the link map $\partial \sigma_{2}: L^{3} \rightarrow S^{4}$. In particular, since the (framed) curve $\sigma_{2}(\gamma)$ is (framed) nullcobordant, the link map $\partial \sigma_{2}$ is fold-cobordant to an (oriented) immersion. That is, there is a compact oriented 4-manifold $W^{4}$ such that $\partial W^{4}=L^{3} \sqcup V^{3}$ and there is a $\Sigma^{1,0}$-map $C:\left(W^{4}, L^{3}, V^{3}\right) \rightarrow\left(S^{4} \times[0,1], S^{4} \times\{0\}, S^{4} \times\{1\}\right)$ such that the restriction $\left.C\right|_{L^{3}}: L^{3} \rightarrow S^{4} \times\{0\}$ is $\partial \sigma_{2}$ and the restriction $\left.C\right|_{V^{3}}: V^{3} \rightarrow S^{4} \times\{1\}$ is an immersion, which we denote by $\partial^{\prime} \sigma_{2}$. It represents an element in $\pi^{s}(3)$ and its image in $\pi^{s}(3) / \mathbb{Z}_{2}$ is independent of the choice of $C$. The obtained element in $\pi^{s}(3) / \mathbb{Z}_{2}$ is $d^{2}\left(\sigma_{2}\right)$.

For future reference we introduce the notation $\sigma_{2}^{*}$ for the map

$$
\sigma_{2}^{*}=\sigma_{2} \underset{L^{3}}{\cup} C: D^{4} \underset{L^{3}}{\cup} W^{4} \rightarrow D_{2}^{5}=D^{5} \underset{S^{4} \times\{0\}}{\cup} S^{4} \times[0,1] .
$$

Note that the two maps coincide on the common part $L^{3}$ of their source manifolds and the gluing can be performed to make $\sigma_{2}^{*}$ smooth.

We shall show that these stable homotopy group elements $d^{1}\left(\sigma_{1}\right), d^{1}\left(\sigma_{2}\right)$, $d^{2}\left(\sigma_{2}\right)$ and other analogously defined ones can be computed from a spectral sequence associated to the classifying spaces of singularities $\left(d^{1}\right.$ and $d^{2}$ are in fact differentials of this spectral sequence). Next we shall describe these

classifying spaces and the spectral sequence, first for the simpler case of prim maps.

\section{$\S 4$ Classifying spaces of cobordisms of singular maps}

Definition. A smooth map $f: M^{n} \rightarrow N^{n+k}$ is called a prim map (prim stands for the abbreviation of projected immersion) if

1) it is the composition of an immersion $g: M \leftrightarrow N \times \mathbb{R}^{1}$ and the projection $\pi: N \times \mathbb{R}^{1} \rightarrow N$, and

2) an orientation is given on the kernels of the differential of $f$.

For maps between manifolds with boundary $f:(M, \partial M) \rightarrow(N, \partial N)$, we shall always require that they should be regular, that is, $f^{-1}(\partial N)=\partial M$ and the map $f$ in a neighbourhood of $\partial M$ can be identified with the direct product $\left.f\right|_{\partial M} \times i d_{[0, \varepsilon)}$ for a suitable positive $\varepsilon$. 
Remark. Note that for a Morin map the kernels of $d f$ form a line bundle ker $d f \rightarrow \Sigma(f)$, where $\Sigma(f)$ is the set of singular points of $f$. The conditions 1 ) and 2) ensure that for a prim map this bundle is orientable (trivial) and an orientation (trivialization) is chosen (the same map $f$ with a different choice of orientation on the kernels is considered to be a different prim map). The converse also holds: if a Morin map $f: M^{n} \rightarrow N^{n+k}$ is equipped with a trivialization of its kernel bundle, then there exists a unique (up to regular homotopy) immersion $g: M \leftrightarrow N \times \mathbb{R}^{1}$ such that $f=\pi \circ g$, where $\pi: N \times \mathbb{R}^{1} \rightarrow N$ is the projection.

Notation. The cobordism group of all prim maps of $n$-dimensional oriented manifolds into $\mathbb{R}^{n+1}$ will be denoted by $\operatorname{Prim}^{S O}(n)$. The analogous cobordism group of prim maps having only (at most) $\Sigma^{1,0}$-singular points (i.e. both the maps and the cobordisms between them are prim fold maps) will be denoted by $\operatorname{Prim}^{S O} \Sigma^{1,0}(n)$. The cobordism group of prim cusp maps will be denoted by $\operatorname{Prim}^{S O} \Sigma^{1,1}(n)$, and the cobordism group of prim $\Sigma^{1_{i}}$-maps will be denoted by $\operatorname{Prim}^{S O} \sum^{1_{i}}(n)$.

Remark. One can define cobordism sets of prim $\Sigma^{1,0}$ and $\Sigma^{1,1}$ (cooriented) maps of $n$-manifolds in arbitrary fixed $(n+1)$-dimensional manifold $N^{n+1}$ (instead of $\mathbb{R}^{n+1}$ ). The obtained sets we denote by $\operatorname{Prim}^{S O} \Sigma^{1,0}(N)$ and $\operatorname{Prim}^{S O} \Sigma^{1,1}(N)$, respectively.

\section{The classifying spaces}

There exist (homotopically unique) spaces $\bar{X} \Sigma^{1,0}$ and $\bar{X} \Sigma^{1,1}$ that represent the functors

$$
\begin{aligned}
& N \longrightarrow \operatorname{Prim}^{S O} \Sigma^{1,0}(N) \text { and } \\
& N \longrightarrow \operatorname{Prim}^{S O} \Sigma^{1,1}(N)
\end{aligned}
$$

in the sense of Brown representability theorem (see $[\mathrm{Sw}]$ ), in particular

$$
\begin{aligned}
& \operatorname{Prim}^{S O} \Sigma_{\Sigma^{1,0}}(N)=\left[N, \bar{X} \Sigma^{1,0}\right] \text { and } \\
& \operatorname{Prim}^{S O} \Sigma^{1,1}(N)=\left[N, \bar{X} \Sigma^{1,1}\right] .
\end{aligned}
$$

We call the spaces $\bar{X} \Sigma^{1,0}$ and $\bar{X} \Sigma^{1,1}$ the classifying spaces for prim fold and prim cusp maps respectively. This type of classifying spaces in a more general setup has been explicitly constructed and investigated earlier, see [Sz2], $\mathrm{Sz} 1],[\mathrm{RSz}$, [Sz4], Te]. 


\section{Key fibrations}

For any space $Y$ we shall denote by $\Gamma Y$ the space

$$
\Omega^{\infty} S^{\infty} Y=\lim _{q \rightarrow \infty} \Omega^{q} S^{q} Y
$$

A crucial observation in the investigation of these classifying spaces is the existence of the so-called key fibrations (see [Sz4]), which in the present cases states that there exist Serre fibrations

$$
\bar{p}_{j}: \bar{X} \Sigma^{1_{j}} \rightarrow \Gamma S^{2 j+1}
$$

of $\bar{X} \Sigma^{1_{j}}$ over $\Gamma S^{2 j+1}$ with fibre $\bar{X} \Sigma^{1_{j-1}}$. In particular, we have

- $\bar{p}_{1}: \bar{X} \Sigma^{1,0} \rightarrow \Gamma S^{3}$ of $\bar{X} \Sigma^{1,0}$ over $\Gamma S^{3}$ with fibre $\Gamma S^{1}$; and

- $\bar{p}_{2}: \bar{X} \Sigma^{1,1} \rightarrow \Gamma S^{5}$ of $\bar{X} \Sigma^{1,1}$ over $\Gamma S^{5}$ with fibre $\bar{X} \Sigma^{1,0}$.

\section{$\S 5$ The spectral sequences}

\section{$\S 5.1$ The first page}

Let us denote by $\bar{X}_{i}$ for $i=-1,0,1,2$ the following spaces:

$$
\bar{X}_{-1}=\text { point } ; \quad \bar{X}_{0}=\Gamma S^{1} ; \quad \bar{X}_{1}=\bar{X} \Sigma^{1,0} ; \quad \bar{X}_{2}=\bar{X} \Sigma^{1,1} .
$$

One can define a spectral sequence with starting page

$$
\bar{E}_{i, j}^{1}=\pi_{i+j+1}\left(\bar{X}_{i}, \bar{X}_{i-1}\right), \quad i=0,1,2, \quad j=0,1, \ldots
$$

and converging to $\pi_{n+1}\left(\bar{X}_{2}\right)=$ Prim ${ }^{S O} \Sigma^{1,1}(n)$.

The existence of Serre fibrations described above implies that

$$
\bar{E}_{i, j}^{1} \cong \pi_{i+j+1}^{S}\left(\Gamma S^{2 i+1}\right)=\pi^{s}(j-i) .
$$

\section{$\S 5.2$ The geometric meaning of the groups and differentials of the spectral sequence}

Let $\bar{X}_{i}$ denote the classifying space of prim $\Sigma^{1_{i}}$-maps, so that $\operatorname{Prim}^{S O} \Sigma^{1_{i}}(n)=$ $\pi_{n+1}\left(\bar{X}_{i}\right)$. The relative versions of the cobordism groups of maps can also be introduced and they will be isomorphic to the corresponding relative homotopy groups: 
Definition. Let $\left(M^{n}, \partial M\right)$ be a compact $n$-manifold with (possibly empty) boundary. Let $f:(M, \partial M) \rightarrow\left(\mathbb{R}_{+}^{n+1}, \mathbb{R}^{n}\right)$ be a prim $\Sigma^{1_{i}}$-map for which $\left.f\right|_{\partial M}: \partial M \rightarrow \mathbb{R}^{n}$ is a $\Sigma^{1_{j}}$ map for some $j \leq i$. Such a map will be called a prim $\left(\Sigma^{1_{i}}, \Sigma^{1_{j}}\right)$-map (recall that we always assume $f$ to be regular in the sense of the definition in $\S 4$ ).

If $f_{0}$ and $f_{1}$ are two prim $\left(\Sigma^{1_{i}}, \Sigma^{1_{j}}\right)$-maps of $n$-manifolds $\left(M_{0}^{n}, \partial M_{0}\right)$ and $\left(M_{1}^{n}, \partial M_{1}\right)$ to $\left(\mathbb{R}_{+}^{n+1}, \mathbb{R}^{n}\right)$, then a cobordism between them is a map $F:\left(N^{n+1}, \partial N\right) \rightarrow\left(\mathbb{R}_{+}^{n+1} \times[0,1], \mathbb{R}^{n} \times[0,1]\right)$ (where $N$ is a compact oriented $(n+1)$-manifold) such that $\partial N=M_{0} \underset{\partial M_{0}}{\cup} Q^{n} \underset{\partial M_{1}}{\cup} M_{1}$ where

a) $Q$ is a cobordism between $\partial M_{0}$ and $-\partial M_{1}$;

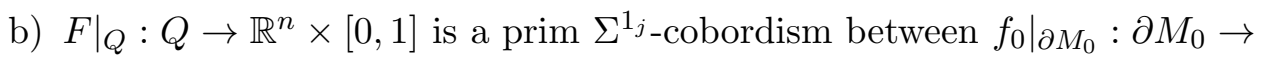
$\mathbb{R}^{n} \times\{0\}$ and $\left.f_{1}\right|_{\partial M_{1}}: \partial M_{1} \rightarrow \mathbb{R}^{n} \times\{1\} ;$

c) $F$ is a prim $\Sigma^{1_{i}}$-map.

Note that both the domain $N$ and the target $\mathbb{R}_{+}^{n+1} \times[0,1]$ of $F$ have "corners": $\partial M_{0} \sqcup \partial M_{1}$ and $\mathbb{R}^{n} \times\{0\} \sqcup \mathbb{R}^{n} \times\{1\}$ respectively. Near the corners $\partial M_{0}$ and $\partial M_{1}$ the map $F$ has to be the direct product of the maps $\left.f_{0}\right|_{\partial M_{0}}$ and $\left.f_{1}\right|_{\partial M_{1}}$ with the identity map of $[0, \varepsilon) \times[0, \varepsilon)$.

The cobordism group of prim $\left(\Sigma^{1_{i}}, \Sigma^{1_{j}}\right)$-maps of oriented $n$-manifolds to $\left(\mathbb{R}_{+}^{n+1}, \mathbb{R}^{n}\right)$ will be denoted by $\operatorname{Prim}^{S O}\left(\Sigma^{1_{i}}, \Sigma^{1_{j}}\right)(n)$.

Analogously to the isomorphism $\operatorname{Prim}^{S O} \Sigma^{1_{i}}(n) \cong \pi_{n+1}\left(\bar{X}_{i}\right)$ one obtains the isomorphism

$$
\operatorname{Prim}^{S O}\left(\Sigma^{1_{i}}, \Sigma^{1_{j}}\right)(n) \cong \pi_{n+1}\left(\bar{X}_{i}, \bar{X}_{j}\right) .
$$

Remark. Note that $\bar{X}_{0}=\Gamma S^{1}$. Indeed, $\Sigma^{1_{0}}=\Sigma^{0}$-maps are non-singular, i.e. immersions, and the classifying space for codimension 1 oriented immersions is known to be $\Gamma S^{1}$ (see e.g. [W]).

The fibration $\bar{p}_{i}:\left(\bar{X}_{i}, \bar{X}_{i-1}\right) \rightarrow\left(\Gamma S^{2 i+1}, *\right)$ induces an isomorphism of homotopy groups $\left(\bar{p}_{i}\right)_{*}: \pi_{n+1}\left(\bar{X}_{i}, \bar{X}_{i-1}\right) \rightarrow \pi_{n+1}\left(\Gamma S^{2 i+1}\right)=\pi^{s}(n-2 i)$. The geometric interpretation of this isomorphism is the following: to the cobordism class of a prim $\Sigma^{1_{i}}$-map $f:(M, \partial M) \rightarrow\left(\mathbb{R}_{+}^{n+1}, \mathbb{R}^{n}\right)$ the mapping $\left(\bar{p}_{i}\right)_{*}$ associates the cobordism class of the framed immersion $\left.f\right|_{\Sigma^{1_{i}(f)}}$ (the framing is described in Appendix 2). Note that in particular this description implies that whenever two prim $\Sigma^{1_{r}}$-maps of $n$-manifolds have framed cobordant images of their $\Sigma^{1_{r}}$-points, then they represent the same element in $\operatorname{Prim}^{S O}\left(\Sigma^{1_{r}}, \Sigma^{1_{r-1}}\right)(n)$. 
The differential

$$
d^{1}: \bar{E}_{i, j}^{1} \cong \pi_{i+j}\left(\bar{X}_{i}, \bar{X}_{i-1}\right) \rightarrow \bar{E}_{i-1, j}^{1} \cong \pi_{i+j-1}\left(\bar{X}_{i-1}, \bar{X}_{i-2}\right)
$$

is simply the boundary homomorphism $\partial$ in the homotopy exact sequence of the triple $\left(\bar{X}_{i}, \bar{X}_{i-1}, \bar{X}_{i-2}\right)$. Composing $\partial$ with the isomorphism $\left(\bar{p}_{i-1}\right)_{*}$ one can see that if $f:\left(M^{n}, \partial M\right) \rightarrow\left(\mathbb{R}_{+}^{n+1}, \mathbb{R}^{n}\right)$ is a prim $\left(\Sigma^{1_{i}}, \Sigma^{1_{i-1}}\right)$-map that represents the cobordism class $[f]=u \in \pi_{n+1}\left(\bar{X}_{i}, \bar{X}_{i-1}\right)=E_{i, n-i}^{1} \cong$ $\pi^{s}(n-2 i)$, then $d^{1}(u) \in \pi^{s}((n-1)-2(i-1))=\pi^{s}(n-2 i+1)$ is represented by the framed immersion $\left.f\right|_{\Sigma^{1-1}\left(\left.f\right|_{\partial M}\right)}$ in $\mathbb{R}^{n}$.

There is an alternative description of $d^{1}$ that we shall use later as well. Let $u \in \pi_{n+1}\left(\bar{X}_{i}, \bar{X}_{i-1}\right)$ and $f$ be a representative of $u$ as above, and let $T$ and $\widetilde{T}$ be the (immersed) tubular neighbourhoods of the top singularity strata $\Sigma^{1_{i}}(f)$ and $f\left(\Sigma^{1_{i}}(f)\right)$ in $M$ and $\mathbb{R}_{+}^{n+1}$, respectively, with the property that $\left.f\right|_{T}$ maps $(T, \partial T)$ to $(\widetilde{T}, \partial \widetilde{T})$. Now $\left.f\right|_{\Sigma^{1_{i-1}\left(\left.f\right|_{\partial T}\right)}}: \Sigma^{1_{i-1}}\left(\left.f\right|_{\partial T}\right) \rightarrow \partial \widetilde{T}$ is a framed immersion into $\partial \widetilde{T}$. Note that the normal framing of $f\left(\Sigma^{1_{i-1}}\left(\left.f\right|_{\partial T}\right)\right)$ inside $\partial \widetilde{T}$ defines a framing of the stable normal bundle of $f\left(\Sigma^{1_{i-1}}\left(\left.f\right|_{\partial T}\right)\right)$ because adding the unique outward-pointing normal vector of $\partial \widetilde{T}$ in $\mathbb{R}_{+}^{n+1}$ one obtains a normal framing in $\mathbb{R}_{+}^{n+1}$. Hence $f\left(\Sigma^{1_{i-1}}\left(\left.f\right|_{\partial T}\right)\right)$ with the given framing represents an element of $\pi^{s}(n-2 i+1)$; this element is $d^{1}(u)$. The fact that these two descriptions of $d^{1}(u)$ yield the same element in $\pi^{s}(n-2 i+1)$ follows from the fact that $f\left(\left.\Sigma^{1_{i-1}} f\right|_{M \backslash T}\right)$ is a stably framed cobordism between the two representatives (here we use Fact 2).

This alternative description of $d^{1}$ actually generalizes to the higher differentials as well (even though here we only consider $d^{1}$ and $d^{2}$ ).

Turning to the differential $d^{2}$, we first give a homotopic description (an expansion of the definition, in fact). Let $u \in \pi^{s}(n-4) \cong \pi_{n+1}\left(\bar{X}_{2}, \bar{X}_{1}\right)=$ $\bar{E}_{2, n-1}^{1}$ be an element such that $d^{1}(u)=0$. Then $u$ represents an element of the page $\bar{E}^{2}$ as well (no differential is going into the groups $\bar{E}_{2, *}^{1}$ ). The class $d^{2}(u) \in E_{0, n}^{2}$ is defined utilizing the boundary homomorphism $\partial: \pi_{n+1}\left(\bar{X}_{2}, \bar{X}_{1}\right) \rightarrow \pi_{n}\left(\bar{X}_{1}\right)$ as follows: since $d^{1}(u)=0$, the class $\partial u \in$ $\pi_{n}\left(\bar{X}_{1}\right)$ vanishes when considered in $\pi_{n}\left(\bar{X}_{1}, \bar{X}_{0}\right)$. Hence there is a class $y$ in $\pi_{n}\left(\bar{X}_{0}\right)$ whose image in $\pi_{n}\left(\bar{X}_{1}\right)$ is $\partial u$. The class $y$ is not unique, but the coset

$$
[y] \in \pi_{n}\left(\bar{X}_{0}\right) / \operatorname{im}\left(\partial^{\prime}: \pi_{n+1}\left(\bar{X}_{1}, \bar{X}_{0}\right) \rightarrow \pi_{n}\left(\bar{X}_{0}\right)\right)=E_{0, n}^{2}
$$

is unique. By definition $d^{2}(u)=[y]$.

Geometrically, if $f:(M, \partial M) \rightarrow\left(\mathbb{R}_{+}^{n+1}, \mathbb{R}^{n}\right)$ represents the class $u \in$ $\pi_{n+1}\left(\bar{X}_{2}, \bar{X}_{1}\right)$, then $d^{1}(u)=0$ means that $\left.f\right|_{\Sigma^{1,0}(\partial f)}: \Sigma^{1,0}(\partial f) \rightarrow \mathbb{R}^{n}$ is 
a null-cobordant framed immersion (recall that $\partial f$ is the restriction $\left.f\right|_{\partial M}$ ). This means that the classifying map $S^{n} \rightarrow \bar{X}_{1}$ of $\partial f$ becomes null-homotopic after composition with $\bar{p}_{1}$, hence the classifying map itself can be deformed into the fiber $\bar{X}_{0}$. Since maps into $\bar{X}_{0}$ classify immersions, this deformation gives a (prim $\Sigma^{1,0_{-}}$)cobordism of the prim $\Sigma^{1,0}$-map $\partial f: \partial M \rightarrow \mathbb{R}^{n}$ to an immersion that we will denote by $g$. The immersion $g$ is not unique, not even its framed cobordism class $[g] \in \pi^{s}(n-1)$ is, but its coset in $\pi^{s}(n-1) /$ im $d^{1}$ is well-defined. This coset is $d^{2}(u)$.

Claim 1. For any $u \in \pi^{s}(n)$

a) $d_{2, n+2}^{1}(u)=d_{2,2}^{1}\left(\sigma_{2}\right) \circ u$

b) $d_{2, n+2}^{2}(u)=d_{2,2}^{2}\left(\sigma_{2}\right) \circ u$

Proof. First we need a description of the composition product in the language of Pontryagin's framed embedded manifolds.

Given $\alpha \in \pi^{s}(m), \beta \in \pi^{s}(n)$ let $\left(M^{m}, U^{p}\right)$ and $\left(N^{n}, V^{m+p}\right)$ be representatives of $\alpha$ and $\beta$, where $M, N$ are manifolds of dimensions $m$ and $n$ immersed to $\mathbb{R}^{m+p}$ and $\mathbb{R}^{n+m+p}$, respectively, $U^{p}$ and $V^{m+p}$ are their framings: $U^{p}=\left(u_{1}, \ldots, u_{p}\right) ; V^{m+p}=\left(v_{1}, \ldots, v_{m+p}\right)$, where $u_{i}, v_{j}$ are linearly independent normal vector fields to $M$ and $N$. These framings identify open tubular neighbourhoods of $M$ and $N$ with $M \times \mathbb{R}^{p}$ and $N \times \mathbb{R}^{m+p}$.

Now we put the framed immersed submanifold $M$ of $\mathbb{R}^{m+p}$ into each fiber of the tubular neighbourhood $N \times \mathbb{R}^{m+p}$. We obtain $N \times M$ as framed immersed manifold in $\mathbb{R}^{n+m+p}$. This is the representative of $\alpha \circ \beta$.

Now we come to the proof of Claim $1 a)$. Let $u$ be an element in $\pi^{s}(n)=\pi_{n+5}\left(\bar{X}_{2}, \bar{X}_{1}\right)$ and let $f:\left(M^{n+4}, \partial M\right) \rightarrow\left(\mathbb{R}_{+}^{n+5}, \mathbb{R}^{n+4}\right)$ be a (relative) prim cusp map that represents a cobordism class corresponding to $u$. The boundary of $f$ is a prim fold map $\partial f=\left.f\right|_{\partial M}: \partial M \rightarrow \mathbb{R}^{n+4}$. Let $\Sigma^{1,0}(\partial f)$ be the manifold of fold points of $\partial f$, and $\partial f\left(\Sigma^{1,0}(\partial f)\right)$ be its image. Since each normal fiber of $\Sigma^{1,0}(\partial f)$ is $\mathbb{R}^{2}$, and it is mapped by $\partial f$ to the corresponding normal fiber $\mathbb{R}^{3}$ of $\partial f\left(\Sigma^{1,0}(\partial f)\right)$ by the Whitney umbrella map $\sigma_{1}$, so $\partial f\left(\Sigma^{1,0}(\partial f)\right)$ has a natural framing (see Appendix 2). Then $d^{1}(u)$ is represented by this framed manifold $\partial f\left(\Sigma^{1,0}(\partial f)\right)$.

Let us choose small tubular neighbourhoods $T$ and $\widetilde{T}$ of $\Sigma^{1,1}(f)$ in $M$ and $f\left(\Sigma^{1,1}(f)\right)$ in $\mathbb{R}_{+}^{n+5}$. $\widetilde{T}$ is immersed into $\mathbb{R}_{+}^{n+5}$, it is a $D^{5}$-bundle over $\Sigma^{1,1}(f)$. Recall that $f$ restricted to $\Sigma^{1,1}(f)$ is an immersion. For simplicity of the description we suppose that it is an embedding. We choose these tubular neighbourhoods $T$ and $\widetilde{T}$ so that $f$ maps $T$ to $\widetilde{T}$ and $\partial T$ to $\partial \widetilde{T}$. Now the map $\left.f\right|_{\partial T}: \partial T \rightarrow \partial \widetilde{T}$ is a fold map. Its singularity is a framed manifold clearly 
representing $d^{1}\left(\sigma_{2}\right) \circ u$. The above described framed manifold $\partial f\left(\Sigma^{1,0}(\partial f)\right)$ (that represents $d^{1}(u)$ ) represents the same framed cobordism class as $\left.f\right|_{\partial T}$ : $\partial T \rightarrow \partial \widetilde{T}$ by the two alternative descriptions of $d^{1}$. Hence $d^{1}(u)=d^{1}\left(\sigma_{2}\right) \circ u$.

The proof of $b$ ) is very similar. As before, for simplicity of the description of $d^{2}$ we suppose that $\left.f\right|_{\Sigma^{1,1}(f)}$ is an embedding rather than an immersion. Let $T$ and $\widetilde{T}$ as above be the tubular neighbourhoods of $\Sigma^{1,1}(f)$ and $f\left(\Sigma^{1,1}(f)\right)$ respectively. Note that (as shown in Appendix 2) $T=$ $\Sigma^{1,1}(f) \times D^{4}, \widetilde{T}=f\left(\Sigma^{1,1}(f)\right) \times D^{5}$ and the map $\left.f\right|_{T}:(T, \partial T) \rightarrow(\widetilde{T}, \partial \widetilde{T})$ is the product map $\left(\left.f\right|_{\Sigma^{1,1}(f)}\right) \times \sigma_{2}$. a map

Recall that in $\S 3$ we defined a map $\sigma_{2}^{*}: D^{4} \cup W^{4} \rightarrow D_{2}^{5}$. Now we define

$$
f^{*}=\left.f\right|_{\Sigma^{1,1}(f)} \times \sigma_{2}^{*}: \Sigma^{1,1}(f) \times\left(D^{4} \underset{L}{\cup} W^{4}\right) \rightarrow f\left(\Sigma^{1,1}(f)\right) \times D_{2}^{5}
$$

We will denote by $T_{2}$ the source manifold of $f^{*}$ and by $\widetilde{T}_{2}$ the target manifold, an enlarged tubular neighbourhood of $f\left(\Sigma^{1,1}(f)\right)$ in $\mathbb{R}_{+}^{n+5}$. Then $\partial f^{*}$ : $\partial T_{2}=\Sigma^{1,1}(f) \times V^{3} \rightarrow \partial \widetilde{T}_{2}=f\left(\Sigma^{1,1}(f)\right) \times S^{4} \times\{1\}$ is an immersion that represents $d^{2}\left(\sigma_{2}\right) \circ u$.

We claim that this immersion can be extended to a proper, regular cusp map $\widehat{f^{*}}$ into the entire $\mathbb{R}_{+}^{n+5}$ without changing the singular set. Indeed, the source manifold of $\partial f^{*}$ has dimension $n+3$, hence the image of $\partial f^{*}$ is an $(n+3)$-dimensional complex (denote it by $K)$, and it can be covered by a small neighbourhood $U$ in $\widetilde{T}_{2}$ of $K$ that deforms onto $K$. By Theorem 2 , there exists a deformation of $U$ (equipped with the outward-pointing normal vector field) within $\mathbb{R}_{+}^{n+5}$ with time derivative nowhere tangent to the image of $U$ that takes $U$ into $\mathbb{R}^{n+4}$ (and the normal vector field into the outwardpointing vector field of $\mathbb{R}^{n+4}$ ). The trace of this deformation glued along $\partial f^{*}$ to $f^{*}$ gives an extension $\widehat{f^{*}}$ whose set of cusp points is the same as that of $f^{*}$ and in particular represents $u$ in $\mathcal{G}$.

This construction shows that $\partial f^{*}$ and $\partial \widehat{f^{*}}$ are cobordant as framed immersions and therefore represent the same element in $\mathcal{G}$; the statement $b$ ) follows since $\partial f^{*}$ represents $d^{2}\left(\sigma_{2}\right) \circ u$ and $\partial \widehat{f^{*}}$ represents $d^{2}(u)$. 


\section{$\S 5.3$ Calculation of the first page of the spectral sequence}

\begin{tabular}{r|c|c|c|l} 
& & & & \\
\hline 3 & $\mathbb{Z}_{24} \stackrel{d_{1,3}^{1}}{\longleftarrow} \mathbb{Z}_{2}$ & $\mathbb{Z}_{2}$ & \\
\hline 2 & $\mathbb{Z}_{2} \stackrel{d_{1,2}^{1}}{\stackrel{y}{=}} \mathbb{Z}_{2} \stackrel{d_{2,2}^{1}}{\longleftarrow}$ & $\mathbb{Z}$ & \\
\hline 1 & $\mathbb{Z}_{2} \stackrel{d_{1}^{1}}{\longleftarrow}$ & $\mathbb{Z}$ & & \\
\hline$j=0$ & $\mathbb{Z}$ & & & \\
\hline & $i=0$ & 1 & 2 &
\end{tabular}

Recall that

$$
\begin{aligned}
\bar{E}_{i, j}^{1} & =\pi_{i+j+1}\left(\bar{X}_{i}, \bar{X}_{i-1}\right) \\
& =\pi_{i+j+1}\left(\Gamma S^{2 i+1}\right)=\pi^{s}(j-i) .
\end{aligned}
$$

Hence on the diagonal $j=i$ we have $\pi^{s}(0)=\mathbb{Z}$ with generator $\iota_{i}$ in $\bar{E}_{i, i}^{1}$ represented by the map $\sigma_{i}$ : $\left(D^{2 i}, S^{2 i-1}\right) \rightarrow\left(D^{2 i+1}, S^{2 i}\right)$ that has an isolated $\Sigma^{1_{i}}$ singularity at the origin. On the line $j=i+t$ we have $\pi^{s}(t)$.

The value $d_{1,1}^{1}\left(\iota_{1}\right)$ is nothing else but $\left[\partial \sigma_{1}\right]=\eta \in \pi^{s}(1)=\mathbb{Z}_{2}$.

By Claim 1 we have $d_{1,2}^{1}(\eta)=d_{1,1}^{1}\left(\iota_{1}\right) \circ \eta=\eta \circ \eta \neq 0$ in $\pi^{s}(2)$ (here and later we refer the reader to [To, Chapter XIV] for the information that we need about the composition product). Hence $d_{1,2}^{1}$ is an isomorphism and it follows that $d_{2,2}^{1}$ is zero (since $d_{1,2}^{1} \circ d_{2,2}^{1}=0$ ). In particular, we have the following lemma:

Lemma 3. The class $d^{1}\left(\iota_{2}\right)$, represented by the image $\sigma_{2}(\gamma)$ of the fold singularity curve on the boundary of the isolated cusp $\sigma_{2}: \mathbb{R}^{4} \rightarrow \mathbb{R}^{5}$, vanishes.

In Appendix 1 we give an independent, elementary proof for this statement.

\section{$\S 5.4$ The second page $\left(\bar{E}_{i, j}^{2}, \bar{d}_{i, j}^{2}\right)$}

The differential $d_{1,3}^{1}: \mathbb{Z}_{2} \rightarrow \mathbb{Z}_{24}$ maps the generator $\eta \circ \eta$ of $\pi^{s}(2)$ to $d_{1,1}^{1}\left(\iota_{1}\right) \circ$ $\eta \circ \eta=\eta \circ \eta \circ \eta$ and that is not zero ([To, Theorem 14.1]). Hence the group 
$E_{0,3}^{2}$ is $\mathbb{Z}_{24} / \mathbb{Z}_{2}=\mathbb{Z}_{12}$.

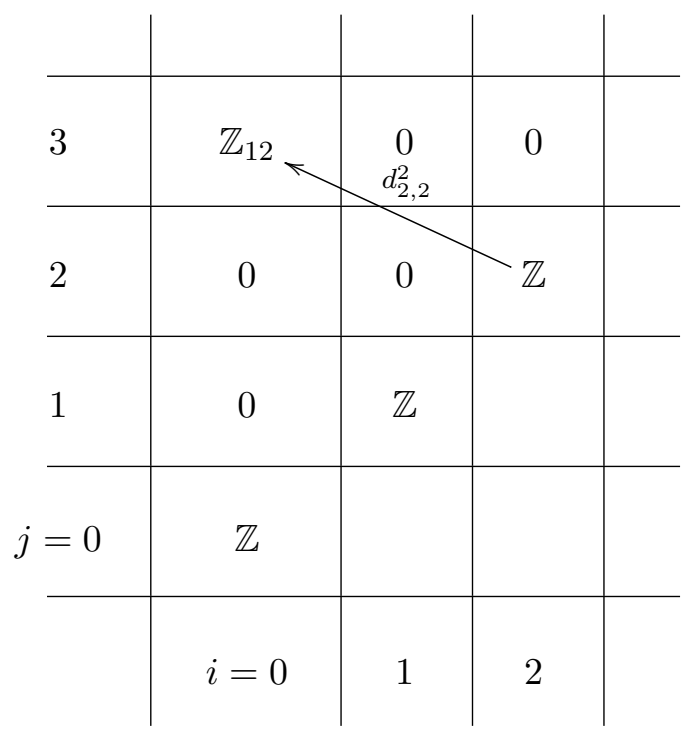

Now we compute the differential $d_{2,2}^{2}: \mathbb{Z} \rightarrow \mathbb{Z}_{12}$. Note that this is precisely the computation of the cobordism class of the framed immersion $\partial^{\prime} \sigma_{2}$ of the 3-manifold $V^{3}$ mentioned in $\S 3$.

Lemma 4. $d_{2,2}^{2}: \mathbb{Z} \rightarrow \mathbb{Z}_{12}$ maps the generator $\iota_{2}$ of $\bar{E}_{2,2}^{2} \cong \mathbb{Z}$ into an element of order 6 .

Proof. $\bar{E}_{2,2}^{1} \cong \pi_{5}\left(\bar{X}_{2}, \bar{X}_{1}\right)=\pi_{5}\left(\Gamma S^{5}\right)=\pi_{5}^{s}\left(S^{5}\right)=\mathbb{Z}$. Since $d_{2,2}^{1}$ is identically zero, $\bar{E}_{2,2}^{2}=\bar{E}_{2,2}^{1}$.

Consider the following commutative diagram with exact row and column:

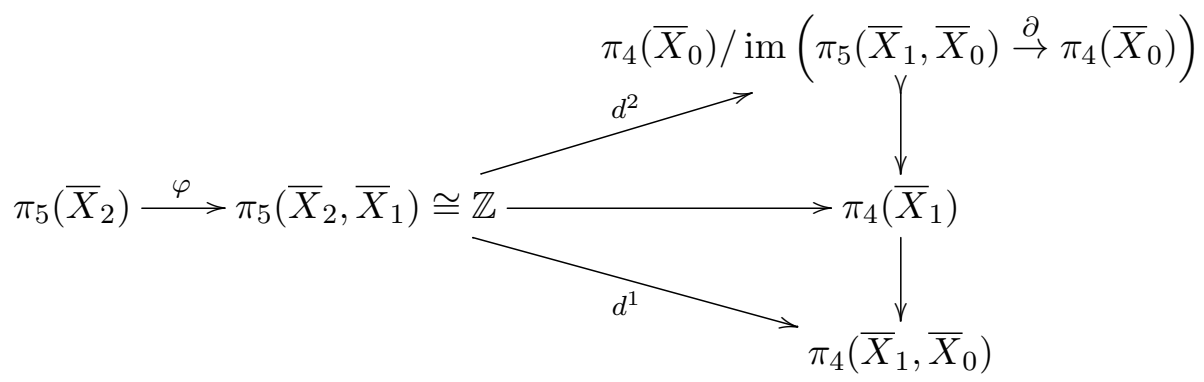

The generator $\iota_{2}$ of $\pi_{5}\left(\bar{X}_{2}, \bar{X}_{1}\right) \cong \mathbb{Z}$ is represented by the cusp map $\sigma_{2}$ : $\left(D^{4}, L^{3}\right) \rightarrow\left(D^{5}, S^{4}\right)$. Simple diagram chasing shows that the order of $d^{2}\left(\iota_{2}\right)$ is equal to the order of Coker $\varphi$. The latter is the minimal positive number 
of cusp points of prim cusp maps of oriented closed 4-manifolds into $\mathbb{R}^{5}$. Indeed, $\varphi$ assigns to the class of a map $f: M^{4} \rightarrow \mathbb{R}^{5}$ the algebraic number of its cusp points. This minimal number of cusps is known to be 6, see [Sz3, Theorem 4].

Corollary. On the 3-torsion part, the differential $d^{2}$ acts as the homomorphism $\alpha_{1}$ (as defined in §2).

\section{$\S 5.5$ The spectral sequence for classifying spaces of arbitrary (not necessarily prim) cusp maps}

There are classifying spaces for the cobordisms of codimension 1 cooriented arbitrary (not necessarily prim) $\Sigma^{1_{i}}$-maps as well. We denote these spaces by $X_{i}=X \Sigma^{1_{i}}$, with the convention that $X_{-1}=*$. Here we will mostly be interested in $X_{0}, X_{1}$ and $X_{2}$. The filtration $X_{-1} \subset X_{0} \subset X_{1} \subset X_{2}$ gives again a spectral sequence with $E_{i, j}^{1}=\pi_{i+j}\left(X_{i}, X_{i-1}\right)$ for $i=0,1,2$, $j=0,1, \ldots$. Analogously to the fibrations $\bar{p}_{i}$ we have fibrations

$$
\begin{gathered}
p_{1}: X_{1} \rightarrow \Gamma T\left(2 \varepsilon^{1} \oplus \gamma^{1}\right)=\Gamma S^{2} \mathbb{R} P^{\infty} \text { with fibre } X_{0}, \text { and } \\
p_{2}: X_{2} \rightarrow \Gamma T\left(3 \varepsilon^{1} \oplus 2 \gamma^{1}\right)=\Gamma S^{3}\left(\mathbb{R} P^{\infty} / \mathbb{R} P^{1}\right) \text { with fibre } X_{1} .
\end{gathered}
$$

Here $\gamma^{1}$ and $\varepsilon^{1}$ are the canonical and the trivial line bundles over $\mathbb{R} P^{\infty}$, respectively, and $T$ stands for Thom space (recall that $\Gamma=\Omega^{\infty} S^{\infty}$ ). Note that $X_{0}=\bar{X}_{0}=\Gamma S^{1}$.

Observe that the base spaces of $p_{i}$ are different from those of $\bar{p}_{i}$. This change is due to the fact that while the normal bundles of the singularity strata for a prim map are trivial and even canonically trivialized (see Appendix 2), for arbitrary cooriented codimension 1 Morin maps they are direct sums of not necessarily trivial line bundles (see [RSz, Theorem 6] and the definition of $G^{S O}$ that precedes it). The bundles $2 \varepsilon^{1} \oplus \gamma^{1}$ and $3 \varepsilon^{1} \oplus 2 \gamma^{1}$ are the universal normal bundles in the target of the fold and cusp strata respectively.

Consequently we have:

Proposition 5. a) $E_{1, j}^{1} \in \mathcal{C}_{2}$.

b) $E_{i, j}^{1} \cong \bar{E}_{i, j}^{1}$ modulo $\mathcal{C}_{2}$ for $i=0,2$.

Proof. a) Since $H_{*}\left(\mathbb{R} P^{\infty} ; \mathbb{Z}_{p}\right)=0$ for $p$ odd, the Serre-Hurewicz theorem implies that $\pi_{*}^{s}\left(\mathbb{R} P^{\infty}\right) \in \mathcal{C}_{2}$ and therefore

$$
E_{1, j}^{1} \cong \pi_{j+1}\left(\Gamma S^{2} \mathbb{R} P^{\infty}\right)=\pi_{j-1}^{s}\left(\mathbb{R} P^{\infty}\right) \in \mathcal{C}_{2}
$$


b) Since the inclusion $S^{2}=\mathbb{R} P^{2} / \mathbb{R} P^{1} \hookrightarrow \mathbb{R} P^{\infty} / \mathbb{R} P^{1}$ induces isomorphism of $\mathbb{Z}_{p}$-homologies (the groups $H_{*}\left(\mathbb{R} P^{\infty} / \mathbb{R} P^{2} ; \mathbb{Z}_{p}\right)$ all vanish) for $p$ odd, we have

$$
E_{2, j}^{1} \cong \pi_{j+1}\left(\Gamma S^{3}\left(\mathbb{R} P^{\infty} / \mathbb{R} P^{1}\right)\right) \cong \pi_{j+2}\left(\Gamma S^{5}\right) \cong \bar{E}_{2, j}^{1}
$$

We also have $X_{0}=\bar{X}_{0}$ and consequently

$$
E_{0, j}^{1} \cong \bar{E}_{0, j}^{1}
$$

\section{§5.6 Computation of the cobordism group of prim fold maps of oriented $n$-manifolds to $\mathbb{R}^{n+1}$}

Theorem 6. a) $\operatorname{Prim}^{S O} \Sigma^{1,0}(n) \underset{\overline{\mathcal{C}_{2}}}{\cong} \pi^{s}(n) \oplus \pi^{s}(n-2)$.

b) $\operatorname{Prim}^{S O^{1,1}}(n) \underset{\mathcal{C}_{\{2,3\}}}{\cong} \pi^{s}(n) \oplus \pi^{s}(n-2) \oplus \pi^{s}(n-4)$.

Proof. We have seen that the spectral sequences computing $\operatorname{Prim}^{S O_{\Sigma}{ }^{1,0}}(n)$ and $\operatorname{Prim}^{S O} \Sigma^{1,1}(n)$ degenerate modulo $\mathcal{C}_{2}$ and modulo $\mathcal{C}_{\{2,3\}}$ respectively, because $d^{1}$ is multiplication by the order 2 element $\eta$ and $d^{2}$ is multiplication by an element of order 6 .

The fact that the cobordism groups $\operatorname{Prim}^{S O} \Sigma^{1,0}(n)$ and $\operatorname{Prim}^{S O} \Sigma^{1,1}(n)$ are direct sums (modulo 2- and 3-primary torsion) can be shown in the same way as in [Sz5, Theorem B]. Namely, the homotopy exact sequence of the fibration $\bar{p}_{1}:\left(\bar{X}_{1}, \bar{X}_{0}\right) \rightarrow \Gamma S^{3}$

$$
\pi_{n+1}\left(\bar{X}_{0}\right) \rightarrow \pi_{n+1}\left(\bar{X}_{1}\right) \stackrel{\left(\bar{p}_{1}\right) *}{\rightarrow} \pi_{n+1}\left(\Gamma S^{3}\right)
$$

has a 2-splitting $s$, that is, there is a homomorphism $s: \pi_{n+1}\left(\Gamma S^{3}\right) \rightarrow$ $\pi_{n+1}\left(\bar{X}_{1}\right)$ such that $\left(\bar{p}_{1}\right)_{*} \circ s$ is the multiplication by 2 . The construction of $s$ goes as follows: choose an immersion $S^{2} \rightarrow \mathbb{R}^{4}$ with normal Euler number 2 . Then its generic projection to $\mathbb{R}^{3}$ will be a map $\psi: S^{2} \rightarrow \mathbb{R}^{3}$ with finitely many Whitney umbrella points that inherit a sign from the orientation of the kernel bundle, and the algebraic number of these points will be 2 (see $[\mathrm{L}]$ ). Now choosing any framed immersion $q: Q^{n-2} \rightarrow \mathbb{R}^{n+1}$ that represents an element $[q]$ in $\pi_{n+1}\left(\Gamma S^{3}\right) \cong \pi^{s}(n-2)$, the framing of $Q$ defines a prim fold map $Q \times S^{2} \stackrel{i d \times \psi}{\rightarrow} Q \times \mathbb{R}^{3} \leftrightarrow \mathbb{R}^{n+1}$. Its class will be $s([q])$. The existence of the 2-splitting map $s$ implies part $a$ ). 
The existence of an analogous 6-splitting of the homotopy exact sequence of the fibration $\bar{p}_{2}:\left(\bar{X}_{2}, \bar{X}_{1}\right) \rightarrow \Gamma S^{5}$ is shown in [Sz5, Lemma 4]. It shows that $\operatorname{Prim}^{S O} \Sigma^{1,1}(n) \underset{\mathcal{C}_{\{2,3\}}}{\cong} \operatorname{Prim}^{S O} \Sigma^{1,1}(n) \oplus \pi^{s}(n-4)$ and together with part a) proves part $b$ ).

Theorem 7. Let $\eta_{n}: \pi^{s}(n) \rightarrow \pi^{s}(n+1)$ be the homomorphism $x \mapsto \eta \circ x$. Then the following sequence is exact:

$$
0 \rightarrow \text { Coker } \eta_{n-1} \rightarrow \operatorname{Prim}^{S O} \Sigma^{1,0}(n) \rightarrow \text { ker } \eta_{n-2} \rightarrow 0
$$

Proof. In the homotopy exact sequence of the pair $\left(\bar{X}_{1}, \bar{X}_{0}\right)$ the boundary homomorphism is the differential $d_{1}$, which by Claim $1 a$ ) is just the corresponding homomorphism $\eta$. The statement follows immediately.

Recall that for any abelian group $G$ we denote by $G_{3}$ its 3-primary part.

Theorem 8. The 3-primary part of $\operatorname{Prim}^{S O} \Sigma^{1,1}(n)$ fits into the short exact sequence

$$
\begin{aligned}
& 0 \rightarrow \text { Coker }\left(\alpha_{1}: \pi^{s}(n-3) \rightarrow \pi^{s}(n)\right)_{3} \oplus\left(\pi^{s}(n-2)\right)_{3} \rightarrow \\
& \rightarrow\left(\operatorname{Prim}^{S O} \Sigma^{1,1}(n)\right)_{3} \rightarrow\left(\operatorname{ker}\left(\alpha_{1}: \pi^{s}(n-4) \rightarrow \pi^{s}(n-1)\right)\right)_{3} \rightarrow 0
\end{aligned}
$$

Proof. The spectral sequence $\bar{E}_{i, j}^{r}$ converges to $\operatorname{Prim}^{S O} \Sigma^{1,1}(n)$ and stabilizes at page 3 . Recall that on the 3-primary part $d^{2}$ can be identified with the homomorphism $\alpha_{1}$. Hence the 3-primary parts $\left(\bar{E}_{i, j}^{3}\right)_{3}$ of the groups $\bar{E}_{i, j}^{3} \cong \bar{E}_{i, j}^{\infty}$ are the following:

$$
\begin{aligned}
& \left(\bar{E}_{0, j}^{3}\right)_{3}=\left(\operatorname{Coker}\left(\alpha_{1}: \pi^{s}(j-3) \rightarrow \pi^{s}(j)\right)\right)_{3} \\
& \left(\bar{E}_{1, j}^{3}\right)_{3}=\left(\pi^{s}(j-1)\right)_{3} \\
& \left(\bar{E}_{2, j}^{3}\right)_{3}=\left(\operatorname{ker}\left(\alpha_{1}:\left(\pi^{s}(j-4) \rightarrow \pi^{s}(j-1)\right)\right)_{3}\right.
\end{aligned}
$$

By general properties of spectral sequences it holds that if we define the groups

$$
\begin{array}{ll}
F_{2, n}=\operatorname{Prim}^{S O} \Sigma^{1,1}(n) & =\pi_{n+1}\left(\bar{X}_{2}\right) \\
F_{1, n}=\operatorname{im}\left(\operatorname{Prim}^{S O} \Sigma^{1,0}(n) \rightarrow \operatorname{Prim}^{S O} \Sigma^{1,1}(n)\right) & =\operatorname{im}\left(\pi_{n+1}\left(\bar{X}_{1}\right) \rightarrow \pi_{n+1}\left(\bar{X}_{2}\right)\right) \\
F_{0, n}=\operatorname{im}\left(\pi^{s}(n) \rightarrow \operatorname{Prim}^{S O} \Sigma^{1,1}(n)\right) & =\operatorname{im}\left(\pi_{n+1}\left(\bar{X}_{0}\right) \rightarrow \pi_{n+1}\left(\bar{X}_{2}\right)\right)
\end{array}
$$


then

$$
\begin{aligned}
F_{2, n} / F_{1, n} & =\bar{E}_{2, n-2}^{\infty} \\
F_{1, n} / F_{0, n} & =\bar{E}_{1, n-1}^{\infty} \\
F_{0, n} & =\bar{E}_{0, n}^{\infty}
\end{aligned}
$$

We will show that the exact sequence $0 \rightarrow\left(F_{0, n}\right)_{3} \rightarrow\left(F_{1, n}\right)_{3} \rightarrow\left(F_{1, n} / F_{0, n}\right)_{3} \rightarrow$ 0 splits and hence $\left(F_{1, n}\right)_{3} \cong\left(F_{0, n}\right)_{3} \oplus\left(F_{1, n} / F_{0, n}\right)_{3}$. Then the exact sequence $0 \rightarrow\left(F_{1, n}\right)_{3} \rightarrow\left(F_{2, n}\right)_{3} \rightarrow\left(F_{2, n} / F_{1, n}\right)_{3} \rightarrow 0$ can be written as $0 \rightarrow\left(F_{0, n}\right)_{3} \oplus\left(F_{1, n} / F_{0, n}\right)_{3} \rightarrow\left(F_{2, n}\right)_{3} \rightarrow\left(F_{2, n} / F_{1, n}\right)_{3} \rightarrow 0$, and substituting $(*)$ gives us the statement of Theorem 8 .

It remains to show that $0 \rightarrow\left(F_{0, n}\right)_{3} \rightarrow\left(F_{1, n}\right)_{3} \rightarrow\left(F_{1, n} / F_{0, n}\right)_{3} \rightarrow 0$ splits. Consider the following commutative diagram:

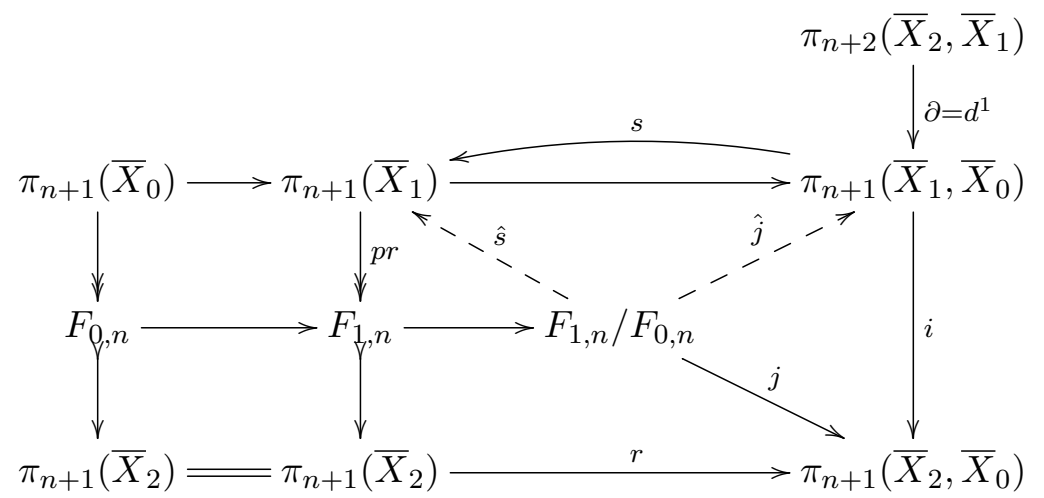

Consider the composition map $F_{1, n} \rightarrow \pi_{n+1}\left(\bar{X}_{2}\right) \rightarrow \pi_{n+1}\left(\bar{X}_{2}, \bar{X}_{0}\right)$. Its kernel is the intersection $\operatorname{ker} r \cap F_{1, n}$; but ker $r$ is the image of $\pi_{n+1}\left(\bar{X}_{0}\right)$, which is $F_{0, n}$. Hence the map $r$ lifts uniquely to a map $j: F_{1, n} / F_{0, n} \rightarrow$ $\pi_{n+1}\left(\bar{X}_{2}, \bar{X}_{0}\right)$. Its image $\operatorname{im} j$ is a subset of $\operatorname{im} i$ due to the commutativity of the right-hand square.

By Claim 1, in the (exact) rightmost column the map $\partial$ (which can be identified with $\left.d^{1}\right)$ acts on $\pi_{n+2}\left(\bar{X}_{2}, \bar{X}_{1}\right) \cong \pi_{n+2}\left(\Gamma S^{5}\right)$ by composition from the left by $\partial\left[\sigma_{2}\right]$, which is zero. Hence the map $i$ is injective. Consequently, the map $j$ can be lifted to a map $\hat{j}: F_{1, n} / F_{0, n} \rightarrow \pi_{n+1}\left(\bar{X}_{1}, \bar{X}_{0}\right)$ and composing it with the 2-splitting $s$ gives us a map $\hat{s}=s \circ \hat{j}: F_{1, n} / F_{0, n} \rightarrow \pi_{n+1}\left(\bar{X}_{1}\right)$ such that $p r \circ \hat{s}$ is a 2 -splitting of the short exact sequence $0 \rightarrow F_{0, n} \rightarrow$ $F_{1, n} \rightarrow F_{1, n} / F_{0, n} \rightarrow 0$. This proves that on the level of 3 -primary parts this extension is trivial, as claimed. 


\section{$\S 5.7$ Computation of the cobordism group of (arbitrary) cusp maps}

Proof of Theorem 11. The natural forgetting map $\bar{E}_{i, j}^{1} \rightarrow E_{i, j}^{1}$ induces a $\mathcal{C}_{2^{-}}$ isomorphism for $i=0,2$, and $E_{1, j}^{1} \in \mathcal{C}_{2}$. Since the $d^{1}$ differential is trivial modulo $\mathcal{C}_{2}$ for both spectral sequences, the map $\bar{E}_{i, j}^{2} \rightarrow E_{i, j}^{2}$ is a $\mathcal{C}_{2^{-}}$ isomorphism for $i=0,2$.

Hence the differential $d^{2}$ restricted to the 3-primary part can be identified in the two spectral sequences, and we obtain that $\left(E_{i, j}^{\infty}\right)_{3}=\left(E_{i, j}^{3}\right)_{3} \cong$ $\left(\bar{E}_{i, j}^{3}\right)_{3}$ for $i=0,2$. The statement of the theorem follows analogously to Theorem 8 .

\section{$\S 6$ Appendix 1: an elementary proof of Lemma 3}

In this Appendix we give an elementary and independent proof of the fact that the curve of folds on the boundary sphere of an isolated cusp map $\sigma_{2}: \mathbb{R}^{4} \rightarrow \mathbb{R}^{5}$ with the natural framing represents the trivial element in $\pi^{s}(1)=\mathbb{Z}_{2}$.

$$
\sigma_{2}: \mathbb{R}^{4} \rightarrow \mathbb{R}^{5}, \sigma_{2}\left(t_{1}, t_{2}, t_{3}, x\right)=\left(t_{1}, t_{2}, t_{3}, t_{1} x+t_{2} x^{2}, t_{3} x+x^{3}\right)
$$

$$
\mathrm{d} \sigma_{2}=\left[\begin{array}{cccc}
1 & 0 & 0 & 0 \\
0 & 1 & 0 & 0 \\
0 & 0 & 1 & 0 \\
x & x^{2} & 0 & t_{1}+2 x t_{2} \\
0 & 0 & x & t_{3}+3 x^{2}
\end{array}\right]
$$

The set of singular points of $\sigma_{2}$ is $\Sigma=\left\{\left(-2 x t_{2}, t_{2},-3 x^{2}, x\right) \mid t_{2}, x \in \mathbb{R}\right\}$, its image is $\tilde{\Sigma}=\sigma_{2}(\Sigma)=\left\{\left(-2 x t_{2}, t_{2},-3 x^{2},-t_{2} x^{2},-2 x^{3}\right) \mid t_{2}, x \in \mathbb{R}\right\}$.

For a point $p \in \mathbb{R}^{4} \backslash \Sigma$ the vector $n(p)=\left(-x\left(t_{3}+3 x^{2}\right),-x^{2}\left(t_{3}+\right.\right.$ $\left.\left.3 x^{2}\right), x\left(t_{1}+2 x t_{2}\right), t_{3}+3 x^{2},-\left(t_{1}+2 x t_{2}\right)\right)$ is non-zero and orthogonal to the columns of $\mathrm{d} \sigma_{2}$, so it is a normal vector of the immersed hypersurface $\sigma_{2}\left(\mathbb{R}^{4} \backslash \Sigma\right) \subset \mathbb{R}^{5}$ at $\sigma_{2}(p)$.

$\tilde{\Sigma} \backslash\{0\}$ is an embedded surface in $\mathbb{R}^{5}$, and it has a canonical framing:

Through each point $p=\left(-2 x t_{2}, t_{2},-3 x^{2}, x\right) \in \Sigma \backslash\{0\}$ we can define a curve $p_{\varepsilon}=\left(-2 x t_{2}, t_{2},-3 x^{2}-\varepsilon^{2}, x+\varepsilon\right)$ such that $p_{0}=p, \frac{\partial p_{\varepsilon}}{\partial \varepsilon}(0)=$ $(0,0,0,1) \in \operatorname{ker} \mathrm{d} \sigma_{2}$, and $\sigma_{2}\left(p_{\varepsilon}\right)=\sigma_{2}\left(p_{-\varepsilon}\right)=q_{\varepsilon^{2}}$, where $q_{\delta}=\left(-2 x t_{2}, t_{2},-3 x^{2}-\right.$ $\left.\delta,-t_{2}\left(x^{2}-\delta\right),-2 x^{3}+2 \delta x\right)$. (Note that by taking this curve for each $p$ we have defined an orientation of the kernel line bundle of $\mathrm{d} \sigma_{2}$.) 
The first vector of the framing is the tangent vector of the image curve $q_{\delta}:$

$$
v_{1}=\frac{\partial q_{\delta}}{\partial \delta}(0)=\left(0,0,-1, t_{2}, 2 x\right) .
$$

Since $\sigma_{2}\left(p_{\varepsilon}\right)=\sigma_{2}\left(p_{-\varepsilon}\right)=q_{\varepsilon^{2}}$, in this point we have defined two normal vectors of $\sigma_{2}\left(\mathbb{R}^{4} \backslash \Sigma\right)$, namely

$$
\begin{aligned}
n\left(p_{ \pm \varepsilon}\right)= & \pm \varepsilon\left(-6 x^{2}-2 \varepsilon^{2},-6 x^{3}-10 x \varepsilon^{2}, 2 x t_{2}, 6 x,-2 t_{2}\right)+ \\
& +\varepsilon^{2}\left(-8 x,-14 x^{2}-2 \varepsilon^{2}, 2 t_{2}, 2,0\right) .
\end{aligned}
$$

The sum and the difference of these vectors are

$$
\begin{aligned}
& n\left(p_{\varepsilon}\right)+n\left(p_{-\varepsilon}\right)=2 \varepsilon^{2}\left(-8 x,-14 x^{2}-2 \varepsilon^{2}, 2 t_{2}, 2,0\right) \text { and } \\
& n\left(p_{\varepsilon}\right)-n\left(p_{-\varepsilon}\right)=2 \varepsilon\left(-6 x^{2}-2 \varepsilon^{2},-6 x^{3}-10 x \varepsilon^{2}, 2 x t_{2}, 6 x,-2 t_{2}\right) .
\end{aligned}
$$

The last two vectors of the framing are the limits of (the directions of) these vectors:

$$
\begin{aligned}
& v_{2}=\left(-3 x^{2},-3 x^{3}, x t_{2}, 3 x,-t_{2}\right) \\
& v_{3}=\left(-4 x,-7 x^{2}, t_{2}, 1,0\right)
\end{aligned}
$$

The following Claim implies that the framed curve $\sigma_{2}(\gamma)$ is null-cobordant (recall that $\gamma=\sigma_{2}^{-1}\left(S^{4}\right) \cap \Sigma$ ).

Claim 2. There is a smooth embedding $F: D^{2}=\left\{\left(t_{2}, x\right) \mid t_{2}^{2}+x^{2} \leq 1\right\} \rightarrow$ $\mathbb{R}^{5}$ and a framing of $F\left(D^{2}\right)$ that extends $\sigma_{2} \circ i$ and the canonical framing of $\tilde{\Sigma} \backslash\{0\}$ restricted to $\sigma_{2} \circ i\left(S^{1}\right)$, where $i: S^{1}=\left\{\left(t_{2}, x\right) \mid t_{2}^{2}+x^{2}=1\right\} \rightarrow \Sigma$, $i\left(t_{2}, x\right)=\left(-2 x t_{2}, t_{2},-3 x^{2}, x\right)$.

Proof. We define such an $F$ and a framing:

$$
\begin{aligned}
F\left(t_{2}, x\right) & =\left(-2 x t_{2}, t_{2},-3 x^{2},-t_{2} x^{2}, 2 x\left(t_{2}^{2}-1\right)\right) \\
v_{1} & =\left(0,0,-1, t_{2}, 2 x\right) \\
v_{2} & =\left(3-3 t_{2}^{2}-6 x^{2},-3 x^{3}, x t_{2}, 3 x,-t_{2}\right) \\
v_{3} & =\left(-4 x,-7 x^{2}, t_{2}, 1,0\right)
\end{aligned}
$$

These are smooth, and in the case $t_{2}^{2}+x^{2}=1$ they coincide with the previously defined map and framing. It is easy to check that $F$ is injective. We need to prove that the differential of $F$ is injective, and the vectors really form a framing, ie. that the partial derivatives of $F$ and $v_{1}, v_{2}, v_{3}$ are linearly 
independent. Equivalently, the following matrix should be non-singular:

$$
\begin{gathered}
M=\left[\begin{array}{ccccc}
-2 x & 1 & 0 & -x^{2} & 4 x t_{2} \\
t_{2} & 0 & 3 x & x t_{2} & 1-t_{2}^{2} \\
0 & 0 & -1 & t_{2} & 2 x \\
3-3 t_{2}^{2}-6 x^{2} & -3 x^{3} & x t_{2} & 3 x & -t_{2} \\
-4 x & -7 x^{2} & t_{2} & 1 & 0
\end{array}\right] \\
\operatorname{det} M=180 x^{8}+568 x^{6} t_{2}^{2}+323 x^{4} t_{2}^{4}+120 x^{6}-197 x^{4} t_{2}^{2}+8 x^{2} t_{2}^{4}+3 t_{2}^{6}+ \\
\quad+51 x^{4}-12 x^{2} t_{2}^{2}-2 t_{2}^{4}+24 x^{2}-2 t_{2}^{2}+3 \\
=50\left(2 x^{2} t_{2}^{2}-x^{2}\right)^{2}+6\left(x t_{2}^{2}-x\right)^{2}+2\left(t_{2}^{3}-t_{2}\right)^{2}+2\left(t_{2}^{2}-1\right)^{2}+ \\
\quad+180 x^{8}+568 x^{6} t_{2}^{2}+123 x^{4} t_{2}^{4}+120 x^{6}+3 x^{4} t_{2}^{2}+2 x^{2} t_{2}^{4}+ \\
\quad+t_{2}^{6}+x^{4}+18 x^{2}+1
\end{gathered}
$$

Therefore $M$ is always non-singular, and the proof is complete.

\section{$\$ 7$ Appendix 2: the natural framing on the image of the manifold formed by the $\Sigma^{1_{r}}$-points of a cooriented prim map}

Let us consider the map

$$
\begin{aligned}
\sigma_{r}:\left(\mathbb{R}^{2 r}, 0\right) & \rightarrow\left(\mathbb{R}^{2 r+1}, 0\right) \\
\left(t_{1}, \ldots, t_{2 r-1}, x\right) & \mapsto\left(t_{1}, \ldots, t_{2 r-1}, z_{1}, z_{2}\right) \\
z_{1} & =t_{1} x+\cdots+t_{r} x^{r} \\
z_{2} & =t_{r+1} x+\cdots+t_{2 r-1} x^{r-1}+x^{r+1}
\end{aligned}
$$

given by the Morin normal form of an isolated $\Sigma^{1_{r}}$ point at 0 . Denote by $\Delta_{r+1}$ the set of $(r+1)$-tuple points, i.e. the points $\left\{p \in \mathbb{R}^{2 r+1}\right.$ : $\sigma_{r}^{-1}(p)$ consists of $r+1$ different points $\}$, and let $\bar{\Delta}$ be the closure of $\Delta_{r+1}$. Then $\bar{\Delta}$ is homeomorphic to an $r$-dimensional ortant $\mathbb{R}_{\angle}^{r}$ with coordinates $u_{1}, \ldots, u_{r}$,

$$
\mathbb{R}_{\angle}^{r}=\left\{\left(u_{1}, \ldots, u_{r}\right): u_{j} \geq 0 \text { for all } j=1, \ldots, r\right\} .
$$

Figure 1 illustrates the case $r=2$. Note that $\mathbb{R}_{\angle}^{r}$ has a natural stratification (the strata are formed by points with the same set of strictly positive coordinates). Moreover a homeomorphism $\varphi: \bar{\Delta} \rightarrow \mathbb{R}_{\angle}^{r}$ can be chosen so that the restriction of $\varphi$ to each stratum is a diffeomorphism. 


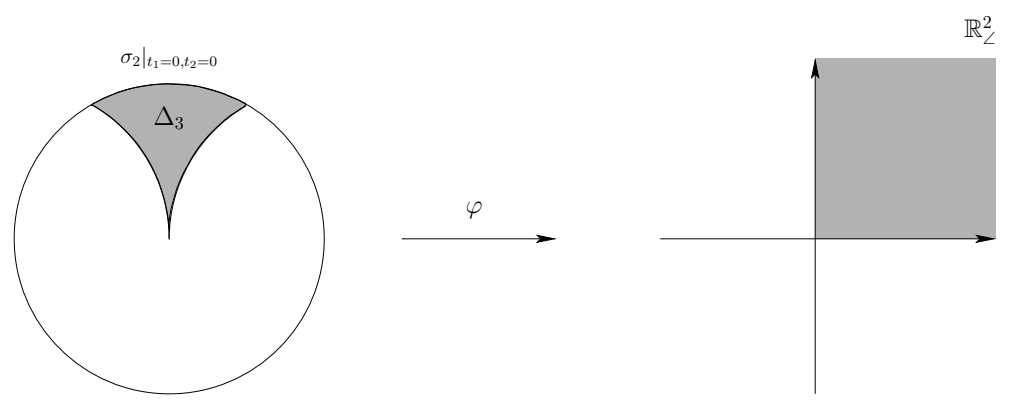

Figure 1: The homeomorphism $\varphi$ for $r=2$.

We want to show that $\bar{\Delta}$ has a (homotopically) canonical parallelization. Clearly any such $\varphi$ does give a parallelization pulled back from $\mathbb{R}_{\angle}^{r}$. But there are many possible choices of $\varphi$ (even up to isotopy). We need to show that there is a canonical (unique up to isotopy) such choice. For this purpose it is enough to show that there is a canonical ordering of the 1-dimensional edges of $\bar{\Delta}$, because if $\varphi$ keeps the ordering of the edges (and it can be chosen to do so), then it is isotopically unique. We shall consider $\sigma_{r}$ as a prim map, namely the projection of the immersion $\left(t_{1}, \ldots, t_{2 r-1}, x\right) \mapsto$ $\left(\sigma_{r}\left(t_{1}, \ldots, t_{2 r-1}, x\right), x\right) \in \mathbb{R}^{2 r+2}$. This gives an ordering of the preimages of multiple points: for an $(r+1)$-tuple point with preimages $a_{1}, \ldots, a_{r+1}$ we assume that the indexing is such that $i<j$ if and only if $x\left(a_{i}\right)<x\left(a_{j}\right)$. If we choose a sequence $\left(q_{n}\right) \in \Delta$ converging to a point $\bar{q} \in \bar{\Delta} \backslash 0$ that lies on a 1-dimensional edge, then the $\sigma_{r}$-preimages of the $(r+1)$-tuple points $\sigma_{r}^{-1}\left(q_{n}\right)=\left\{a_{1}^{(n)}, \ldots, a_{r+1}^{(n)}\right\}$ degenerate in the limit in the sense that there is an integer $s, 1 \leq s \leq r+1$ and there are two different points $\underline{a}$ and $\bar{a}$ with $x(\underline{a})<x(\bar{a})$ such that $\lim _{n \rightarrow \infty} a_{i}^{(n)}=\underline{a}$ for $i=1, \ldots, s$ while $\lim _{n \rightarrow \infty} a_{i}^{(n)}=\bar{a}$ for $i=s+1, \ldots, r+1$. Hence to each edge we can associate an integer $s$, $1 \leq s \leq r+1$, and thus we obtain an ordering of the edges of $\bar{\Delta}$. We choose the map $\varphi$ in such a way that it preserves this ordering of the edges.

Note that any automorphism of $\sigma_{r}$ as a prim map keeps the ordering of the edges of $\bar{\Delta}$. We call a pair $(\alpha, \beta)$ of germs of diffeomorphisms $\alpha$ : $\left(\mathbb{R}^{r}, 0\right) \rightarrow\left(\mathbb{R}^{r}, 0\right)$ and $\beta:\left(\mathbb{R}^{r+1}, 0\right) \rightarrow\left(\mathbb{R}^{r+1}, 0\right)$ a prim automorphism of the prim germ $\sigma_{r}=\pi \circ \hat{\sigma}_{r}:\left(\mathbb{R}^{r}, 0\right) \rightarrow\left(\mathbb{R}^{r+2}, 0\right) \rightarrow\left(\mathbb{R}^{r+1}, 0\right)$ if in addition to being an automorphism of $\sigma_{r}$ (that is, $\sigma_{r} \circ \alpha=\beta \circ \sigma_{r}$ ) it preserves the selected orientation of $\operatorname{ker} \mathrm{d} \sigma_{r}(0)$, i.e. $\frac{\partial \alpha}{\partial x}>0$ (recall that $\left.\frac{\partial \sigma_{r}}{\partial x}(0)=0\right)$.

Let now $g: M^{n} \leftrightarrow \mathbb{R}^{n+2}$ be an immersion, where $M$ is a compact oriented $n$-dimensional manifold. Let $f$ be the prim map $f=\pi \circ g$, with $\pi: \mathbb{R}^{n+2} \rightarrow \mathbb{R}^{n+1}$ the projection that omits the last coordinate $x_{n+2}$ in 
$\mathbb{R}^{n+2}$. Suppose that $f$ is a $\Sigma^{1_{r}}$-map, that is, it has no $\Sigma^{1_{j}}$ points for $j>r$. The set of $\Sigma^{1_{r}}$-points of $f$ will be denoted by $\Sigma^{1_{r}}(f)$, or $\Sigma$ for brevity. It is a submanifold of $M$ of dimension $n-2 r$. Its image $f\left(\Sigma^{1_{r}}(f)\right)$ (denoted by $\tilde{\Sigma}$ ) is an immersed submanifold in $\mathbb{R}^{n+1}$ and has codimension $2 r+1$. We claim that $\tilde{\Sigma}$ has a natural normal framing, unique up to homotopy. To simplify the presentation of the proof, we will suppose that $\tilde{\Sigma}$ is embedded into $\mathbb{R}^{n+1}$ (as it locally is; in general, we would need to consider only the preimages of multiple points that are close to the singular points in the source manifold).

There is a unique (up to isotopy) embedding $\theta: \Sigma \times(-\varepsilon, \varepsilon) \hookrightarrow \mathbb{R}^{n+1}$ (for some small positive number $\varepsilon$ ) such that

- $\theta(x, 0)=f(x)$ for all $x \in \Sigma$,

- $\theta(x, t)$ is an $(r+1)$-tuple point of $f$ for all $0<t<\varepsilon$, and

Choose any $t^{*} \in(0, \varepsilon)$ and denote by $\Sigma^{*}$ the image set $\theta\left(\Sigma \times\left\{t^{*}\right\}\right)$. Clearly it is enough to give a canonical normal framing of $\Sigma^{*}$. Let $\Delta_{r+1}(f)$ denote the set of $(r+1)$-tuple points of $f$ that lie in a tubular neighbourhood of $\Sigma$. There is a fibration $\Delta_{r+1}(f) \rightarrow \Sigma^{*}$ with fiber $\bar{\Delta}$.

The normal bundle of $\Sigma^{*}$ in $\mathbb{R}^{n+1}$ is the sum of its normal bundle in $\Delta_{r+1}(f)$ and the restriction of the normal bundle of $\Delta_{r+1}(f)$ in $\mathbb{R}^{n+1}$ to $\Sigma^{*}$. The latter bundle is trivial, because it is the sum of the trivial normal line bundles of the $(r+1)$ non-singular branches of $f$ that intersect at the points of $\Delta_{r+1}(f)$ (and these branches have a canonical ordering by the last coordinate of $g$ ). The former bundle is trivial because there exists a canonical parallelization of $\bar{\Delta}$ that is (homotopically) invariant under the prim automorphisms of $\sigma_{r}$.

In other words, the target representation (assigning the map $\beta$ to the automorphism $(\alpha, \beta)$ ) of the automorphism group (or, more precisely, the maximal compact subgroup thereof) is trivial. Since we consider positive codimensional stable maps, the target representation uniquely determines the source representation (on the dense set of non-singular, non-multiple points it clearly does) and therefore the source representation is also trivial. That is, there is a canonical framing of the singular strata in the source as well.

\section{References}

[L] G. Lippner: Multiple-point formulas and their applications, https://www . cs.elte.hu/math/phd_th/lippner.pdf 
[M] B. Morin: Formes canoniques des singularités d'une application différentiable, Comptes Rendus 260 (1965), pp. 5662-5665, pp. 6502-6506.

[RSz] R. Rimányi, A. Szűcs: Pontrjagin-Thom-type construction for maps with singularities, Topology 37 (6), pp. 1177-1191.

[RS] C. Rourke, B. Sanderson: The Compression Theorem I, Geometry \& Topology 5 (2001), pp. 399-429.

[S] J. P. Serre: Groupes d'homotopie et classes de groupes abéliens. Ann. of Math. (2) $\mathbf{5 8}$ (1953), pp. 258-294.

[Sw] R. M. Switzer: Algebraic topology - homotopy and homology, reprint of the 1975 original, Classics in Mathematics, Springer-Verlag, Berlin, 2002

[Sz1] A. Szücs: Cobordism of maps with simplest singularities, Topology Symposium, Siegen 1979 (Proc. Sympos., Univ. Siegen, Siegen, 1979), pp. 223-244., Lecture Notes in Math. 788, Springer, Berlin, 1980

[Sz2] A. Szücs: Analogue of the Thom space for mappings with singularity of type $\Sigma^{1}$, (Russian) Mat. SB. (N.S) 108(150) (1979) no.3. pp. 433-456., 478., English translation: Math. USSR-Sb. 36 (1979) no. 3. pp. 405-426. (1980)

[Sz3] A. Szücs: On the singularities of hyperplane projections of immersions, Bull. London Math. Soc. 32 (2000), 364-374.

[Sz4] A. Szűcs: Cobordism of singular maps, Geom. Topol. 12, no. 4 (2008), 23792452.

[Sz5] A. Szücs: On the cobordism groups of cooriented, codimension one Morin maps, J. Singul. 4 (2012), 196-205.

[Te] T. Terpai: Fibration of classifying spaces in the cobordism theory of singular maps, Tr. Mat. Inst. Steklova 267 (2009), Osobennosti i Prilozheniya 280287. Translation in Proc. Steklov Inst. Math. 267, no. 1 (2009), 270-277.

[To] H. Toda: Composition methods in the homotopy groups of spheres, Ann. Math. Studies 49 Princeton (1962).

[W] R. Wells: Cobordism groups of immersions, Topology 5 (1966), pp. 281-294. 
Authors addresses:

Csaba Nagy

Department of Analysis

Eötvös Loránd University (ELTE)

Budapest, Pázmány P. sétány I/C

H-1117 Hungary

e-mail: csaba224@freemail.hu

András Szűcs

Department of Analysis

Eötvös Loránd University (ELTE)

Budapest, Pázmány P. sétány I/C

H-1117 Hungary

e-mail: szucs@cs.elte.hu

Tamás Terpai

A. Rényi Mathematical Institute

of the Hungarian Academy of Sciences

Budapest, Reáltanoda u. 13-15

H-1053 Hungary

e-mail: terpai@renyi.mta.hu 